\title{
Panorama do ensino de odontogeriatria nas universidades públicas brasileiras
}

\author{
Overview of Geriatric Dentistry Education in Public Universities in Brazil
}

Panorama de la enseñanza de odontogeriatría en las universidades públicas brasileiras

\section{Recebido: 04/05/2016 \\ Aprovado: 11/08/2016 \\ Publicado: 01/09/2016}

\author{
María del Rosario Ruiz Núñez ${ }^{1}$ \\ Heloisa Godói ${ }^{2}$ \\ Ana Lúcia Schaeffer Ferreira de Mello ${ }^{3}$
}

Este estudo tem como objetivo apresentar o panorama do ensino de Odontogeriatria nos cursos de graduação em Odontologia das universidades públicas no Brasil. Analisou-se, por meio de estatística descritiva, a oferta desta disciplina, mediante pesquisa nos sítios eletrônicos das universidades públicas brasileiras, bem como suas caracteristicas. 0 estudo constatou que menos da metade dos cursos investigados oferecem a disciplina de Odontogeriatria e, quando o fazem, enfatizam o ensino teórico com conteúdos básicos ao cuidado da saúde bucal de pessoas idosas. As informações estavam disponíveis nos sítios eletrônicos de grande parte das universidades, porém nem todas as características principais foram apresentadas. É relevante qualificar a oferta desta disciplina em termos metodológicos e de conteúdo, para que futuros cirurgiões-dentistas estejam aptos a cuidar da saúde bucal deste grupo populacional.

Descritores: Idoso; Educação em Odontologia; Envelhecimento; Ensino.

This study aims at presenting an overview of geriatric dentistry teaching in Brazilian public dentistry undergraduate courses. The availability of this subject and its characteristics were analyzed with the use of descriptive statistics, through searching in official websites, as well as their features. The study has found that less than half of the investigated courses offer geriatric dentistry as a subject, and that they emphasize theoretical basic contents than the actual practice of oral health care with the elderly. The official websites of most universities had the information needed; however, the main characteristics of the courses were not present in all of them. It is important to qualify the subject in the courses where it is available, taking into account both methodology and content, in order for soon-to-be dental surgeons to be apt to care for the oral health of these group of people.

Descriptors: Elderly; Dental Education; Aging; Education.

Este estudio tuve como objetivo presentar el panorama de la enseñanza de Odontogeriatria en las facultades de Odontología de universidades públicas en el Brasil. Se Analizó, por medio de estadística descriptiva, la oferta de este curso, mediante los sitios electrónicos de las universidades públicas brasileras. Se encontró que menos de la mitad de las facultades investigadas ofrecen el curso de Odontogeriatria y, cuando lo hacen, enfatizan la enseñanza teórica con contenidos básicos sobre el cuidado de la salud bucal de personas ancianas. Las informaciones estaban disponibles en los sitios electrónicos de gran parte de las universidades, sin embargo no todas las características principales fueron presentadas. Es relevante la oferta de este curso, metodológicamente y de contenido, para que futuros cirujanos-dentistas estén aptos para cuidar de la salud bucal de este grupo poblacional.

Descriptores: Anciano; Educación en Odontología; Envejecimiento; Enseñanza.

1 Cirurgiã Dentista. Especialista em Reabilitação Oral. Mestranda em Odontologia em Saúde Coletiva pela Universidade Federal de Santa Catarina (UFSC). É membro do Grupo de Pesquisa Políticas, Ações e Serviços de Saúde Bucal e do Grupo de Pesquisa Observatório de Gestão do Cuidado à Saúde Bucal. charoruizn@gmail.com

${ }^{2}$ Cirurgiã Dentista. Especialista em Saúde da Família. Mestre em Odontologia em Saúde Coletiva. Doutoranda em Odontologia em Saúde Coletiva pela UFSC. heloisagodoi@gmail.com

3 Cirurgiã Dentista. Mestre em Odontologia. Doutora em Odontologia em Saúde Coletiva. Doutora em Enfermagem. Pós Doutora em Enfermagem em Administração e Gerência do Cuidado em Enfermagem e Saúde. Professor Adjunto na UFSC. ana.mello@ufsc.br 


\section{INTRODUÇÃO}

O aumento proporcional do contingente de idosos na população tem ocasionado um volume maior de problemas crônicodegenerativos de saúde ${ }^{1}$, os quais necessitam de respostas adequadas, em termos de ações, e serviços de saúde preparados para lidar com estas novas demandas sociossanitárias ${ }^{2-4}$.

0 processo fisiológico de envelhecimento humano, gradual e previsível, pode ser visto como questão de saúde pública relevante, devido às alterações funcionais, associadas ou não à ocorrência de doenças sistêmicas ${ }^{4,5}$ e bucais $^{6}$. Estas transformações, em conjunto com aspectos socioeconômicos e psicossociais, tornam o grupo populacional idoso mais vulnerável e se traduz em um grande desafio para governos e organizações sociais, incluindo as áreas da educação e da formação acadêmica ${ }^{5,7,8}$.Assim, o perfil epidemiológico atual da população brasileira passa a exigir dos profissionais de saúde novas práticas de cuidado voltadas à população idosa $^{3,7,9}$.

No âmbito da Odontologia, a ênfase a este cuidado ocorre por meio da área de conhecimento denominada Odontogeriatria ou Odontologia Geriátrica. Esta assume a atenção à saúde de idosos, estudando fenômenos decorrentes do envelhecimento, bem como a promoção da saúde, o diagnóstico, a prevenção e o tratamento de enfermidades bucais e do sistema estomatognático do idoso ${ }^{10,11}$. Alguns estudos $^{12-15}$ resgatam o processo histórico de inserção de conteúdos de geriatria e gerontologia nos currículos de graduação em Odontologia em alguns países do mundo. Em geral, países latino-americanos apresentam poucos estudos relacionados ao ensino de Odontogeriatria quando comparados às universidades européias e norte-americanas ${ }^{16}$. Entretanto, mesmo estudos realizados em países como Alemanha, Suíça e Áustria demonstram que conteúdos referentes à saúde do idoso são ministrados em disciplinas de prótese dentária e carecem de disciplina específica com conteúdos de geriatria na matriz curricular dos cursos de graduação de
Odontologia ${ }^{17,18}$.No Brasil, há relatos do ano de 1996 sobre a primeira escola de Odontologia a inserir em seu currículo uma disciplina voltada à saúde bucal de idosos, a qual pertencia à Universidade Estadual de Maringá e denominava-se Estomatogeriatria ${ }^{13}$.

A qualificação de profissionais que já atuam como cirurgiões-dentistas e a formação de graduandos em odontologia para a atenção e assistência odontológica aos idosos é defendida na literatura ${ }^{11,19}$. Os profissionais de odontologia precisam estar aptos a realizar uma abordagem adequada à saúde bucal da população idosa ${ }^{19}$. Assim, a inserção de conteúdos sobre Geriatria e Gerontologia no currículo dos cursos de graduação torna-se indispensável para a formação dos cirurgiõesdentistas contemporâneos ${ }^{18}$.

No entanto, a Odontogeriatria ou Odontologia Geriátrica continua sendo negligenciada nos currículos como disciplina específica e apresenta-se como conteúdo pulverizado juntamente a outras áreas de conhecimento. A ênfase aos conteúdos inerentes a atenção à saúde do idoso ainda é restrita, quando comparada àquela direcionada aos conteúdos relacionados às crianças e aos adolescentes ${ }^{15}$. Este fato pode ser explicado também pelo histórico das políticas públicas no Brasil, as quais por muito tempo mantiveram o foco da atenção à saúde bucal restrito a crianças em idade escolar, excluindo da atenção odontológica pública as demais faixas etárias ${ }^{15,20}$. Desta forma, a defesa pela inclusão de disciplina específica evita que os conteúdos sobre saúde do idoso e envelhecimento sejam diluídos em outras disciplinas ou ministrados de forma parcial e possibilita contato mais aproximado do aluno com o idoso ${ }^{21}$.

Observa-se carência de estudos nacionais que busquem retratar a situação do ensino de Geriatria e Gerontologia no âmbito da formação em nível de graduação em Odontologia. Constata-se, nos poucos estudos publicados no cenário nacional, reduzido número de universidades brasileiras que 
ofertam o ensino de Odontogeriatria como disciplina curricular ${ }^{19}$.

Desta forma, este estudo objetiva apresentar o panorama do ensino de Odontogeriatria nos cursos de graduação em Odontologia das universidades públicas brasileiras, por meio do levantamento do número de cursos de graduação em instituições públicas no país que ofertam a disciplina de Odontogeriatria em seus currículos.

\section{MÉTODO}

Trata-se de um estudo de caráter exploratório, transversal, descritivo com abordagem quantitativa.

0 universo do estudo compreendeu todos os cursos de Odontologia habilitados e em operação nas universidades públicas do Brasil que possuíam em sua matriz curricular a disciplina de Odontogeriatria e/ou disciplina correlata.

Para a seleção da amostra, investigou-se previamente o total de universidades públicas que apresentavam curso de graduação em Odontologia no sítio eletrônico de acesso público do Ministério da Educação. Segundo informações coletadas, entre Janeiro e Abril de 2015, existem no Brasil 57 cursos de graduação em Odontologia em universidades públicas.

Para seleção e inserção das instituições na amostra de pesquisa, foram considerados como critérios de inclusão: ser curso de Odontologia devidamente reconhecido pelo Ministério de Educação; estar ativo e com pelo menos uma turma formada; possuir a disciplina de Odontogeriatria (ou nomenclatura similar); e apresentar toda a informação das variáveis estudadas necessárias via web. Foram excluídos os cursos cujas informações via sítio eletrônico encontravam-se incompletas ou desatualizadas.

Após a seleção da amostra buscou-se caracterizar as seguintes variáveis: período em que a disciplina de Odontogeriatria era ofertada aos alunos de graduação, caráter em que era oferecida (obrigatória ou optativa), natureza que possui (teórica, prática ou teórico-prática) e descrição da ementa com conteúdos ministrados.

Os dados foram organizados em planilhas do software Microsoft Excel ${ }^{\circledR}$ e analisados segundo estatística descritiva, considerando-se a distribuição e frequência dos mesmos.

\section{RESULTADOS}

De acordo com o Ministério da Educação, no ano 2015, o número de cursos de graduação em Odontologia, em atividade plena, ofertados por universidades públicas brasileiras compreendem o total de 57 graduações, inseridas em 47 instituições públicas, visto que algumas universidades apresentam mais que um curso de Odontologia, em diferentes faculdades ou em períodos (noturno/diurno) distintos.

Dentre as 57 entidades analisadas, 45 $(78,9 \%)$ disponibilizam as informações sobre matriz curricular em sítio eletrônico da web. No âmbito dos 45 cursos de graduação, 21 $(46,7 \%)$ ofertam a disciplina de Odontogeriatria, ou equivalente, em sua matriz curricular (Tabela 1).

Em relação ao caráter em que as disciplinas de Odontogeriatria são ofertadas nas 21 instituições analisadas, 11 (52,4\%) apresentam o caráter obrigatório e $10(47,6 \%)$ instituições a ofertam de forma eletiva (Tabela 1). Com respeito à natureza da disciplina, 6 $(28,57 \%)$ universidades oferecem de modo exclusivamente teórico e $6(28,57 \%)$ de forma teórico-práticas. Em 9 (42,86\%) outros cursos esta informação não foi especificada. Já no que concerne ao período em que a disciplina é ministrada, grande parte das instituições $(90,5 \%)$ o fazem entre o sexto e o décimo períodos.

Quanto ao plano de ensino e à descrição da ementa da disciplina de Odontogeriatria, ou equivalente, $10(47,6 \%)$ cursos de graduação em Odontologia disponibilizam o conteúdo da ementa em seu sítio eletrônico e somente 8 $(38,1 \%)$ apresentam o plano de ensino na web. 
Tabela 1. Disciplinas de Odontogeriatria ofertadas em cursos de graduação de Universidades Públicas Brasileiras, segundo características estudadas. Brasil, 2015.

\begin{tabular}{lcc}
\hline Característica & N & \% \\
\hline Caráter da disciplina & & \\
$\quad$ Obrigatório & 11 & 52,4 \\
$\quad$ Optativo & 10 & 47,6 \\
Natureza da disciplina & 6 & 28,6 \\
$\quad$ Teórica & 6 & 28,6 \\
$\quad$ Teórica e prática & 9 & 42,8 \\
$\quad$ Não especifica & & \\
Período de oferta & 2 & 9,5 \\
$\quad$ Entre 10 e 50 período & 19 & 90,5 \\
$\quad$ Entre 60 e 10 período & & \\
Disponibilizam ementa via web & 10 & 47,6 \\
$\quad$ Sim & 11 & 52,4 \\
$\quad$ Não & & \\
Disponibilizam plano de ensino & 8 & 30,1 \\
$\quad$ Sim & 13 & 61,9 \\
$\quad$ Não & & \\
\hline
\end{tabular}

Ao analisar os 8 planos de ensino disponíveis, os conteúdos mais freqüentemente abordados foram: Envelhecimento populacional, (descrito nos planos de ensino de 5 disciplinas); Condição da saúde bucal do idoso, (temas levantados em 7 planos); e, Aspectos gerais da saúde do idoso(assunto indicado em 8 planos de ensino).

\section{DISCUSSÃO}

0 crescimento da população idosa, de 60 anos ou mais, é uma realidade tanto em países desenvolvidos como em países em desenvolvimento $7,11,14,16-18,22,23$. No Brasil, segundo a Pesquisa Nacional por Amostra de Domicílios (PNAD), realizado em 2013, os idosos somaram 25,4 milhões de pessoas, o que corresponde a $13,1 \%$ do total dos brasileiros para o mesmo ano ${ }^{24}$.

0 incremento no número da população idosa no país tem relação com as melhorias nas condições de vida e saúde que ocorreram nas últimas décadas. Com o aumento da expectativa de vida, é importante que as diferentes áreas da saúde estudem mais sobre os processos relacionados ao envelhecimento com a finalidade de promover saúde e prevenir doenças nessa faixa etária ${ }^{25}$. Assim, é importante a adoção de estratégias e medidas que leve em conta as necessidades das pessoas idosas e promova a saúde e o bem-estar na senilidade ${ }^{26}$, incluindo a formação de profissionais de saúde bem preparados para cuidar da saúde da população que envelhece ${ }^{18}$.

No Brasil, e em outros países da América do Sul, a Odontologia Geriátrica é uma disciplina relativamente nova nos cursos de graduação em Odontologia. Nos Estados Unidos da América, essa disciplina reporta aos anos de 1970, quando se iniciou o interesse por criar programas de ensino voltados à saúde dos idosos. Em 1985, 100\% das escolas de Odontologia já relatavam possuir disciplina de Odontologia geriátrica em seus currículos, embora com uma variação considerável de conteúdos ${ }^{12,27}$.

No Canadá, estudo realizado por Ethinger22, mostra que a partir de 1967 o Conselho sobre Saúde Pública da Associação Odontológica Canadense identificou limitações no cuidado à saúde bucal de idosos. Assim, naquela época, iniciava-se o ensino ao cuidado do idoso, porém de maneira simbólica, inserido 
em outras disciplinas, também afirma que, com as mudanças que ocorreram no ensino odontológico desde a última publicação sobre o perfil do ensino de Odontogeriatria até o ano de 2010, a maioria dos currículos de Odontologia canadenses já apresenta cursos de Odontologia geriátrica, porém sem evidências científicas comprovadas.

Nas universidades do Chile, entre os anos 2000 e 2013, 7 universidades apresentavam a disciplina de Odontologia Geriátrica formalmente, ou seja, em caráter obrigatório, nas 19 graduações de Odontologia analisadas no referido artigo, o que corresponde a $37 \%$ dos cursos estudados; por sua vez, as graduações que ainda não possuíam a disciplina em seus currículos o fariam em um futuro próximo, com vistas a atender à demanda demográfica atual ${ }^{16}$.

Já em cursos de graduação na Índia, país que apresenta o mais alto número de escolas de Odontologia em todo o mundo - 291 até o ano de 2010 -, a disciplina de Odontologia Geriátrica não costuma estar presente nos currículos atuais ${ }^{23}$. A inserção de conteúdos sobre o cuidado à saúde do idoso nas escolas de Odontologia da Índia, assim como a atuação de profissionais de saúde de maneira multidisciplinar, também precisam ser vistos como conteúdos e atividades indispensáveis à formação do cirurgião-dentista, visto a dimensão do número de pessoas idosas que carecem destes serviços nos dias atuais ${ }^{23}$. Situação semelhante ocorre no Irã, onde as graduações em Odontologia dispõem de um programa de 6 anos de formação com disciplinas de ciências básicas, pré-clínicas e clínicas, porém a Odontogeriatria não está presente na matriz curricular destes cursos, sendo oferecida apenas como curso de pósgraduação 28 .

No presente estudo, foram identificados 57 cursos de graduação de Odontologia em universidades públicas brasileiras, segundo a página eletrônica de acesso livre do Ministério da Educação. Dentre estes cursos, foi possível buscar informações sobre grade curricular em $78,9 \%$ (45) das instituições, por meio de website institucional. Destes, pouco menos da metade dos cursos, $46,7 \%$ (21), apresentavam a disciplina de Odontogeriatria. Desta forma, constata-se a fragilidade no ensino de Odontogeriatria também na realidade brasileira, considerando-se a eminente transição demográfica atual em todo o mundo, a qual gera um grande número de idosos que apresentam considerável demanda por cuidados de saúde, incluindo-se cuidados odontológicos $7,18,23,29$.

De acordo com as diretrizes curriculares nacionais do curso de graduação em Odontologia, a formação do cirurgiãodentista deve contemplar o sistema de saúde vigente no país e proporcionar ao profissional de Odontologia o desenvolvimento de aptidões compatíveis ao cuidado integral à saúde das pessoas, em todos os seus ciclos de vida. 0 futuro cirurgião-dentista deve estar apto a compreender a realidade social, cultural e econômica do seu meio, dirigindo sua atuação para a transformação da realidade em benefício da sociedade, bem como, ser capaz de pensar criticamente, analisar os problemas correntes que afligem a população e buscar soluções para os mesmos 30 .

Sendo assim, a atuação do cirurgiãodentista brasileiro deve ser marcada pelo conhecimento da realidade da população, a qual requer, neste momento, maior conhecimento do indivíduo idoso e o processo de envelhecimento ${ }^{31}$. 0 profissional de saúde, em consonância com o sistema de saúde público brasileiro, precisa estar capacitado para atuar diariamente em contato com indivíduos idosos, o que possibilita conhecer as necessidades de saúde desta faixa etária da população e, a partir disso, intervir de forma mais humanizada, crítica e reflexiva ${ }^{32}$.

Os achados deste estudo não destoam dos encontrados de outra pesquisa ${ }^{13}$, que analisaram o ensino de Odontogeriatria no Estado da Bahia e constataram que a implementação desta disciplina ainda encontra-se em fase inicial. Os autores ratificam a necessidade em transmitir os conhecimentos relativos a essa área de forma 
segregada às disciplinas de prótese dentária e enfatizam a adequação das estruturas curriculares com o intuito de preparar, de forma mais evidente, os futuros profissionais de odontologia para a resolução das demandas da população idosa.

Referente à natureza da disciplina de Odontogeriatria, este estudo encontrou iguais porcentagens entre teórica e teórico-prática $(28,57 \%)$. Dentre as disciplinas descritas como teórico-práticas, cinco apresentam caráter obrigatório e dispensam em média 30,4 horas para o eixo teórico e 63,6 horas para as atividades práticas. Nessas disciplinas, a carga horária teórica mínima é 30 horas e a máxima de 60 horas, enquanto a prática variou de 60 a 120 horas. No entanto, ao considerar o universo de 21 cursos de graduação que já apresentam a disciplina de Odontogeriatria, estes valores tornam-se diminutos e exprimem a fragilidade da disciplina no âmbito nacional. Para haver melhor formação dos estudantes de graduação em Odontologia algumas modificações nos currículos se fazem necessárias, como a maior valorização e realização de atividades práticas ${ }^{18}$. 0 intuito seria proporcionar aos alunos não apenas contato com o conteúdo teórico, mas que possam também colocá-lo em prática e, assim, desenvolverem melhor sua formação e serem capazes de resolver, criticamente, uma gama variada de problemas. 0 ensino de Odontologia Geriátrica apenas didaticamente, sem experiência clínica, tornar-se-ia insuficiente ${ }^{22}$.

Os alunos de Odontologia devem desenvolver competências, habilidades e atitudes que contribuam a um melhor manejo da pessoa idosa. De acordo com a Academia Europeia de Odontogeriatria, além das competências principais peculiares ao ensino de Odontologia, a disciplina de Odontogeriatria apresenta um caráter interdisciplinar e complementar a outras disciplinas. Desta forma, o ensino deve se preocupar em enfatizar o caráter interdisciplinar e proporcionar aos alunos a convivência com outros especialistas que estão envolvidos com o cuidado da pessoa idosa ${ }^{18}$.
0 ensino interprofissional na área da saúde possibilita ao aluno de graduação desenvolver e compreender um trabalho em conjunto, como forma de viabilizar o melhor manejo do paciente. Esta experiência, durante a formação, permite que os estudantes adquiram habilidades e competências para responder às necessidades da população a partir do trabalho em equipe e possibilita reconhecer tanto os limites da ação individual como explorar a atuação das diferentes profissões envolvidas com o cuidado do paciente ${ }^{33}$.

Do mesmo modo, a inserção de atividades de treinamento clínico e pré-clínico em diferentes locais como hospitais, unidades geriátricas, diretamente em comunidades ou por meio de atendimento on site (nos domicílios, em instituições de longa permanência ou hospitais) favorecem $o$ aprendizado e tornam os estudantes aptos tanto a planejarem o cuidado de pessoas mais idosas, sejam elas dependentes funcionalmente ou não, bem como adquirir habilidades para assisti-las, as quais não seriam factíveis sem este contato ${ }^{18}$.

A atuação de cirurgiões-dentistas em ambientes diferentes ao consultório odontológico não é exclusiva à Odontogeriatria, visto que o ser humano exige um cuidado multidisciplinar próprio de sua complexidade. 0 cuidado à saúde em si, ao compreender o indivíduo em toda sua integridade física, psíquica e social, exige uma atuação integrada entre profissionais da área da saúde. A presença de cirurgiões-dentistas em equipes com diferentes especialidades torna-se cada vez mais frequente e pode levar à formação de profissionais mais comprometidos com a realidade de saúde e com sua transformação social ${ }^{34}$. 0 treinamento de futuros cirurgiões-dentistas em ambiente hospitalar junto a pacientes idosos, assim como em unidades de terapia intensiva, atualmente transforma-se em realidade ${ }^{34}$.

Neste estudo, ao buscarem-se as temáticas abordadas pelos cursos, verificou-se que apenas $38,1 \%$ dos cursos de Odontologia 
analisados disponibilizam os planos de ensino via $w e b$ e ao averiguá-los constatou-se que os conteúdos da disciplina de Odontogeriatria expostos apresentam como temas mais frequentes aqueles considerados básicos para o cuidado à saúde bucal do idoso: envelhecimento populacional, aspectos gerais da saúde bucal do idoso e condições da saúde bucal do idoso. 0 aumento da população idosa, a formação do aluno tem que ser plena em diferentes termos; processo de ensino e aprendizagem deve ter uma ativa participação do aluno35. Assim, é importante que os currículos sejam flexíveis e ajustados à realidade.

É importante destacar que a não identificação das informações via web não significa necessariamente que os cursos de Odontologia de universidades públicas não trabalhem com tal conteúdo em outras disciplinas ou que desconsiderem o valor deste tema na formação profissional. Trata-se de uma limitação do estudo.

Referente ao período em que a disciplina é ministrada, 90,5\% das instituições pesquisadas oferta a disciplina de Odontogeriatria entre o sexto e décimo período. Existe concordância com os cursos de Odontologia que ofertam a disciplina de Odontogeriatria nos últimos semestres, pois os alunos apresentariam maior segurança por disporem de melhor experiência na prática clínica, no cuidado e no atendimento ao paciente idoso ${ }^{13}$.

Os achados deste estudo corroboram com evidências internacionais ${ }^{18}$, os quais relatam que a disciplina de Odontogeriatria deve ser oferecida nos cursos de graduação e requer uma revisão profunda nos currículos atuais. A base para o currículo disciplinar em Odontogeriatria perpassa pela compreensão das características orais, médicas, psicológicas, físicas e mentais de pacientes idosos e deve estar pautado no cuidado centrado no paciente e na abordagem interdisciplinar, além de ultrapassar o aspecto eminentemente teórico e ser complementado com experiências práticas em diferentes situações ${ }^{18}$.
Como constatado em outros estudos $11,13,14,16-18,23,25,28,29$, a população idosa em constante crescimento carece de profissionais qualificados para o seu atendimento e a aquisição desses conhecimentos durante o período dos cursos de graduação em Odontologia torna-se oportuno e permite o adequado treinamento dos cirurgiões-dentistas para o cuidado a esta população.

\section{CONCLUSÃO}

$\mathrm{O}$ estudo constatou que menos da metade dos cursos investigados oferecem a disciplina de Odontogeriatria ou equivalente e, quando o fazem, enfatizam o ensino teórico com conteúdos básicos ao cuidado da saúde bucal de pessoas idosas. As informações estavam disponíveis nos sítios eletrônicos de grande parte das universidades, porém nem todas as características principais foram apresentadas.

Há necessidade de se qualificar a oferta desta disciplina, em termos metodológicos e de conteúdo, para que os futuros cirurgiõesdentistas estejam aptos a cuidar da saúde bucal dos idosos que estão em crescente demanda. Nesse sentido, a inclusão da disciplina de Odontogeriatria no currículo dos cursos pode ser uma possibilidade para uma melhor formação na área.

0 artigo pretendeu discutir sobre 0 cenário dos cursos de Odontologia das universidades públicas brasileiras e traz contribuições tanto para o meio acadêmico quanto ao setor dos serviços públicos voltados a esta população, com vistas a melhorar o cuidado à saúde bucal dos idosos.

\section{REFERÊNCIAS}

1. Scommegna P. Noncommunicable diseases among older adults in low-and middle-income countries. Today's Research on Aging 2012; (26):1-7.

2. World Health Organization. Good health adds life to years: Global Brief for World Health Day 2012. Geneva: WHO. 2012; 2012. 28.

3. Veras R. Envelhecimento populacional e as informações de saúde do PNAD: demandas e 
desafios contemporâneos. Cadernos de Saúde Pública 2009; 43(3):458-54.

4. Beard JR, Bloom, DE. Towards a comprehensive public health response to population ageing. The Lancet 2015; 385(9968):658-61.

5. Tavares DMS, Ribeiro KB, Silva CC, Montanholi LL. Ensino de gerontologia e geriatria: uma necessidade para os acadêmicos da área da saúde da Universidade Federal do Triângulo Mineiro?. Ciência, Cuidado e Saúde. 2008; 7(4):537-45.

6. Petersen PE, Yamamoto, T. Improving the oral health of older people: the approach of the WHO global oral health programme. Community Dentistry and Oral Epidemiology 2005; 33(2):81-92.

7. Camararo AA. (Org). Novo regime demográfico: uma nova relação entre população e desenvolvimento? Rio de Janeiro: IPEA, 2014:658.

8. Moraes E. Atenção à saúde de idoso: aspectos conceituais. Brasília: Organização Pan-Americana da Saúde. 2012, 98p.

9. Veras R. A urgente e imperiosa modificação no cuidado à saúde da pessoa idosa. Revista Brasileira de Geriatria e Gerontologia 2015; 18(1):5-6.

10. Rosa LB, Zuccolotto MCC, Bataglion C, Coronatto EAS. Odontogeriatria - a saúde bucal na terceira idade. Revista da Faculdade de Odontologia - UPF 2008; 13(2):82-6.

11. Ettinger RL. 30-year review of a geriatric dentistry teaching programme. Gerodontology 2010; 29(2):e1252-60.

12. Mohammad R, Preshaw PM, Ettinger RL. Current status of predoctoral geriatric education in U.S. dental schools. Journal of Dental Education 2003; 67(5):509-14.

13. Francisco KMS, Dias PN, Casotti CA, Uemura TF, Filho DLG. Ensino da Odontogeriatria nos cursos de graduação no Estado da Bahia. Clínica e Pesquisa em Odontologia - UNITAU 2014; 6(1):28-35.

14. Macedo IAB, Oliveira CCC, Freitas MMD, Nunes MAR. Odontogeriatria e necessidade curricular-relato de acadêmicos da UNIT-SE.
Odontologia Clínico-Científica 2002; 1(2):10922.

15. Lima JCS. Projetos políticos pedagógicos dos cursos de odontologia do Rio Grande do Norte e a perspectiva da atenção idosa. [disssertação de mestrado]. Rio Grande do Norte(RN): Univerdade Federal do Rio Grande do Norte/UFRN;2011. 75p.

16. León S, Bustos FA, Ettinger RL, Giacaman RA. Geriatric dentistry content in the curriculum of the dental schools in Chile. Gerodontology 2014:1-7.

17. Nitschke I, Kunze J, Reiber T, Sobota BAJ. Development of undergraduate gerodontology courses in Austria, Switzerland, and Germany from 2004-2009. Journal of Dental Education 2013; 77(5):630-9.

18. Kossioni A, Vanobbergen J, Newton J, Muller F, Heath R. European College of Gerodontology: undergraduate curriculum guidelines in Gerodontology. Gerodontology 2009; 26(3):165-71.

19. Saintrain MVL, Souza EHA, Caldas Júnior AF. Ensino da odontogeriatria nas faculdades de odontologia do sul e centro-oeste do Brasil : situação atual e perspectivas. Revista Odonto Ciência 2006; 21(53):270-7.

20. Nickel DA, Lima FG, Silva BB. Modelos assistenciais em saúde bucal no Brasil. Cad. Saúde Pública 2008; 24(2):241-6.

21. Santos SSC. O ensino da enfermagem gerontogeriatrica e a complexidade. Revista da Escola de Enfermagem-USP. 2006; 40(2):22835.

22. Ettinger RL. The development of geriatric dental education programs in Canada: An Update. Journal of the Canadian Dental Association 2010; 76(1):1-4.

23. Thomas S. The need for geriatric dental education in India: the geriatric health challenges of the millennium. International Dental Journal 2013; 63(3):130-6.

24. IBGE. Síntese de indicadores sociais: Uma análise das condições de vida da população brasileira. Rio de Janeiro: IBGE, 2013, 337p.

25. Bezerra FC, Almeida MI, Nóbrega-Therrien SM. Estudo sobre envelhecimento no Brasil. 
Revista Brasilera de Geriatria e Gerontologia 2012; 15(1):155-67.

26. World Health Organization. Envelhecimento ativo: Uma política em Saúde. Organización Pan-America de Salud-OPAS; OMS. Brasília: OPAS, 2015;(1).

27. Ettinger RL. Geriatric dentistry in the USA: looking back 30 years. Gerodontology 2014; 31:161-5.

28. Mir APB. Need for geriatric dentistry training programs in Iran. Journal of Dental Education 2013; 77(1):113-7.

29. Hatami B, Ahmady AE, Khoshnevisan MH, Lando HA. Dental student perceived barriers in geriatric dental care active involvement. Oral Health Dental Management 2014; 13(3):675-9. 30. Brasil. Conselho Nacional de Educação. Câmara de Educação Superior. Resolução CNE/CES n.3, de 19 de fevereiro de 2002, Institui Diretrizes Curriculares Nacionais do Curso de Graduação em Odontologia. Diário Oficial da União, Brasília, DF, 4 mar. 2002. Seção 1, p.10.

31. Moimaz SAS, Garbin CAS, Lolli LF, Dossi AP, Nayme JGR. . Percepção de acadêmicos de odontologia sobre o envelhecimento. Revista de Odontologia da UNESP 2010; 39(4):227-31. 32. Casate JC, Corrêa AK. A humanização do cuidado na formação dos profissionais de saúde nos cursos de graduação. Revista da Escola de Enfermagem da USP. 2011; 46(1):219-26.
33. Aguilar-da-Silva RH, Scapin LT, Batista NA. Avaliação da formação interprofissional no ensino superior em saúde: aspectos da colaboração e do trabalho em equipe. Revista da Avaliação da Educação Superior 2011; 16(1):167-84.

34. Araújo RJG, Vinagre NPL, Sampaio JMS. Avaliação sobre a participação de cirurgiõesdentistas em equipes de assistência ao paciente. Acta Scientiarum Health Sciences 2009; 31(2):153-7.

35. Garbin CAS, Saliba NA, Moimaz SAS, Santos KT. O papel das universidades na formação de profissionais na área de saúde. Revista da ABENO 2006; 6(1):6-10.

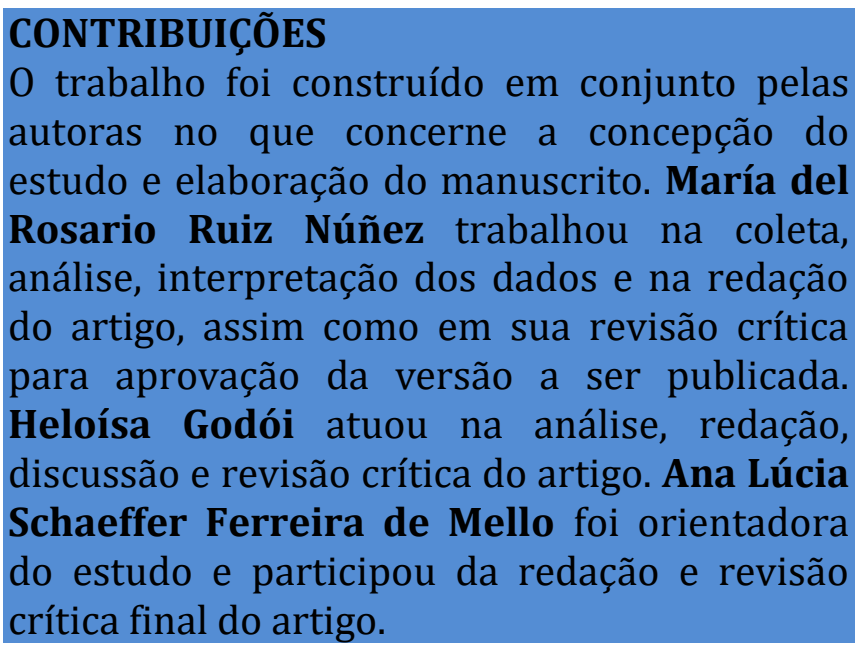

\section{Como citar este artigo (Vancouver):}

Núñez MRR, Godói H, Mello ALSF. Panorama do ensino de odontogeriatria nas universidades públicas brasileiras. REFACS [Internet]. 2016 [citado em (inserir dia, mês e ano de acesso)]; 4(3):237-245. Disponível em: (link de acesso). DOI: 10.18554/refacs.v4i3.1776.

Como citar este artigo (ABNT):

NÚÑEZ, M.R.R.; GODÓI, H.; MELLO, A.L.S.F.Panorama do ensino de odontogeriatria nas universidades públicas brasileiras. REFACS, Uberaba, MG, v. 4, n. 3, p. 237-245, 2016. Disponível em: (link de acesso). Acesso em: (inserir dia, mês e ano de acesso). DOI: 10.18554/refacs.v4i3.1776.

\section{Como citar este artigo (APA):}

Núñez, M.R.R., Godói, H. \& Mello, A.L.S.F.(2016). Panorama do ensino de odontogeriatria nas universidades públicas brasileiras. REFACS, 4(3), 237-245. Recuperado em (inserir dia, mês e ano de acesso)(inserir link de acesso). DOI: 10.18554/refacs.v4i3.1776. 